\title{
Experimental Study on PAT System for Long-Distance Laser Communications Between Fixed-Wing Aircrafts
}

\author{
Yao $\mathrm{LEI}^{1^{*}}$, Xiaoming $\mathrm{LI}^{2}$, and Lizhong $\mathrm{ZHANG}^{3}$ \\ ${ }^{1}$ School of Mechanical Engineering and Automation, Fuzhou University, Fuzhou 350116, China \\ ${ }^{2}$ National and Local Joint Engineering Research Center of Space Optoelectronics Technology, Changchun University of \\ Science and Technology, Changchun 130000, China \\ ${ }^{3}$ Institute of Space Optoelectronic Technology, Changchun University of Science and Technology, Changchun 130000, \\ China \\ *Corresponding author: Yao LEI \\ E-mail: yaolei@fzu.edu.cn
}

\begin{abstract}
Free-space laser communication is characterized by high communication speed, strong anti-jamming ability, high confidentiality, and flexible configuration. In this paper, a pointing, acquisition, and tracking (PAT) system based on a two-stage (i.e., coarse and fine) composite tracking mechanism is proposed to solve the optical axis alignment problem, which is common in free-space laser communications. The acquisition probability of the PAT system is ensured by designing two tracking modules, a coarse tracking module which combines passive damping with active suppression and a fine tracking module based on an electromagnetic galvanometer. Both modules are combined by using a dynamic scanning mechanism based on the gyroscope signal. Finally, a free-space laser communication test with a long range and a high speed is conducted by two fixed-wing Y12 aircrafts equipped with the proposed PAT system. Experimental results show that the coarse tracking precision of the airborne PAT system is $10 \mu \mathrm{rad}(1 \sigma)$, and the fine tracking precision is $8 \mu \mathrm{rad}(1 \sigma)$ during flights which are much improved as compared with the indoor tests. This indicates that the system can achieve a high precision for PAT during high-speed and long-range laser communications in the free-space. This also verifies the tracking capability and the environmental adaptability of the proposed laser communication PAT system.
\end{abstract}

Keywords: PAT system; laser communications; airborne platform; coarse tracking error; fine tracking error

Citation: Yao LEI, Xiaoming LI, and Lizhong ZHANG, "Experimental Study on PAT System for Long-Distance Laser Communications Between Fixed-Wing Aircrafts," Photonic Sensors, 2019, 9(2): 170-178.

\section{Introduction}

An aircraft furnished with an array of sensors can act as a flexible reconnaissance platform, enabling it to continuously acquire information about the ground, sea, and free-space which can be disseminated to ground stations over high-speed links $[1,2]$. Also, an aircraft can act as a node in a communication network, functioning as a relay station to expand the coverage of terrestrial and ocean surface networks or to relay information between aerial and ground units. With the continuous advancement of the detection technology, an increase in the detector resolution and a rapid growth of data volumes have increased the need for the development of communication channels with

Received: 16 July 2018 / Revised: 4 October 2018

(C) The Author(s) 2018. This article is published with open access at Springerlink.com DOI: 10.1007/s13320-018-0522-9

Article type: Regular 
high communication bandwidths [3]. To address these challenges, the laser communication technology in the free-space, with its advantages of high transmission rate, high confidentiality, and strong anti-interference ability, has recently been proposed as an efficient method for information dissemination on aerial platforms. The development of the free-space laser communication technology can significantly improve the data transmission capability of information networks.

Till date, there have been a few significant laser communication tests on airborne platforms which have been carried out by various research groups across the world. The TT Company of USA conducted an aircraft-to-ground station laser communication test to achieve a $1 \mathrm{Gbps}$ data rate at a flight height of $1.1 \mathrm{~km}$ while communicating over the $20 \mathrm{~km}-30 \mathrm{~km}$ range [4-6]. In another test carried out by Massachusetts Institute of Technology (Massachusetts, USA) in 2009, the researchers were able to achieve a data transmission rate of $2.5 \mathrm{Gbps}$ at a flight height of $3.657 \mathrm{~km}$, while communicating over a distance of $25 \mathrm{~km}$ [7]. In 2011, a joint experiment by TT Company and Air Force Research Laboratory (AFRL) of USA performed an aircraft-to-aircraft laser communication test to achieve a data rate of $2.5 \mathrm{Gbps}$ for a communication range of $94 \mathrm{~km}-132 \mathrm{~km}$ [8]. Changchun University of Science and Technology in 2011 carried out a series of tests to achieve a data rate of $1.5 \mathrm{Gbps}$ at a flight height of $600 \mathrm{~m}-800 \mathrm{~m}$, while communicating over a distance of $17.5 \mathrm{~km}$ with a bit error rate of $10^{-6}[9,10]$.

As a high-speed motion platform, the communication range of the aircraft is relatively wide and complied with the rapid position and attitude change in real time, or even strong vibration. The atmosphere boundary layer also brings a great difficulty to the wireless laser communications, which may seriously restrict the communication speed and distance. This is because, when the laser passes through the atmosphere, the power of the laser beam received by the detector fluctuates and is attenuated due to the rapid changes because of the mentioned factors [11, 12]. This affects the detection precision and has a negative impact on the laser communication system. Although the power fluctuation caused by the atmospheric turbulence is relatively weak in inter-aircraft laser communications, the speckle effect caused by atmospheric turbulence dampens the tracking precision [13]. Moreover, when the aircraft is flying at a high speed, the violent vibrations of the aircraft, large low-frequency disturbances, and rapid and random posture changes can also cause the precision of optical axis alignment to be lower than the minimum precision required for reliable laser communications and open-loop pointing [14]. Therefore, in order to ensure a high-precision optical axis alignment in free-space laser communications, we must employ a pointing, acquisition, and tracking (PAT) system to counter the effects of vibration and the disturbance of the aircraft platform. Otherwise, the communication link cannot be established at all.

\section{PAT system}

\subsection{System structure}

The posture of the aircraft can have a wide range of values, or even with a high frequency vibration. Therefore, a PAT system needs not only a wide tracking bandwidth or high tracking precision, but also a large tracking range to realize high-precision optical axis alignment for free-space laser communications. To achieve this goal, a two-stage (coarse, fine) composite tracking scheme is adopted in this study (see Fig. 1).

In the first stage, the coarse tracking module is employed in the structure. Due to a relatively low tracking precision and narrow tracking bandwidth, the coarse tracking module is going to bring the target within the viewing scope of the fine tracking system. In the second stage, the fine tracking module is activated with a narrow tracking range, 
high precision, and wide tracking bandwidth to satisfy the requirement of high precision tracking. The composition of the proposed free-space laser communication PAT system is also demonstrated in Fig. 1. The system mainly consists of a coarse tracking turntable, an optical base station which is composed of the fine tracking module and other components, and the PAT control unit.

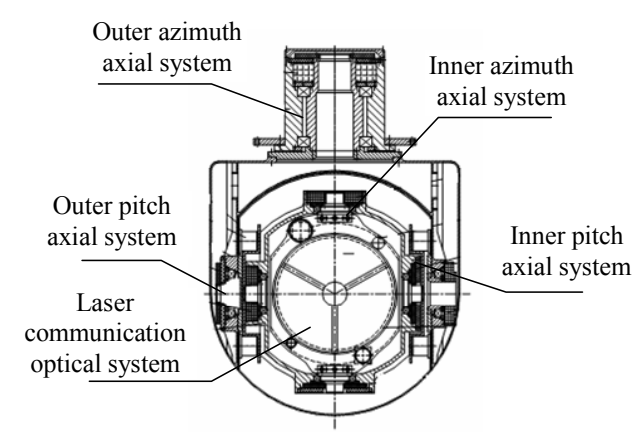

Fig. 1 Composition of the PAT system.

The fine tracking module consists of a charge coupled device (CCD) spot detection unit, a fine tracking control unit, and a galvanometer. Usually, the key errors that affect the precision of the fine tracking system include (1) actuator error, (2) spot detection error, (3) dynamic hysteresis error, and (4) errors due to residual platform vibration. Two techniques employed to improve the tracking precision of the fine tracking system and achieve the high-precision spot detection are CCD sub-pixel subdivision and automatic integration time adjustment. Additionally, a certain camera in the random read-out of interest window (ROI mode) is also applied with techniques such pixel synthesis to achieve an output frame rate of 2000 frames per second (fps).

The scanning trajectory will cease to be an ideal one when the posture of the platform changes. For the experiments in this paper, a one-dimensional angular motion simulation platform is applied to simulate the posture changes (sine change) of the platform. It is observed that a scanning trajectory based on the turntable angle will not be suitable in the presence of interference. This is because of two factors: (1) the scanning angle is based on the inertial space, and (2) the initial angle of scanning is determined by the angle at the time of pointing. Since the angle of scanning is reported in the geodetic coordinate system, it is necessary to perform a coordinate transformation on the scanning angle. Meanwhile, it is also necessary to add the compensation due to the gyro drift when generating the instruction $[15,16]$. These can be represented in (1) and (2) as

$$
\begin{gathered}
\theta_{\mathrm{az}-\mathrm{scan}}=\left[v_{\mathrm{az}-\mathrm{scan}}+R_{\mathrm{az}}\left(v_{g \text {-az }}+v_{\text {gshift-az }}\right)\right] \times t_{\text {scan }} \\
\theta_{\text {el-scan }}=\left[v_{\text {el-scan }}+R_{\mathrm{el}}\left(v_{g \text {-el }}+v_{\text {gshift-el }}\right)\right] \times t_{\text {scan }}
\end{gathered}
$$

where $v_{\mathrm{az}-\mathrm{scan}}$ and $v_{\mathrm{el}-\mathrm{scan}}$ are the designed scanning speeds in the directions of the azimuth and the pitch; $v_{g \text {-az }}$ and $v_{\text {-el }}$ are the velocities of the rate of change of the gyro in the directions of the azimuth and pitch; $v_{\text {gshift-az }}$ and $v_{\text {gshift-el }}$ are the speed shifts of the gyro in the azimuth and pitch directions; $R_{\mathrm{az}}$ and $R_{\mathrm{el}}$ are the coefficients which arise due to the coordinate transformation (coupling is ignored in small ranges); $t_{\text {scan }}$ is the scanning interval.

\subsection{Key parameters of the system}

The operating procedure of the system can be divided into three stages: (1) initial pointing, (2) rapid acquisition, and (3) accurate tracking. Only after these three stages are complete, the PAT system can start the tracking accurately. The required coarse tracking precision is $50 \mu \mathrm{rad}(1 \sigma)$, and the required fine tracking precision is $8 \mu \mathrm{rad}(1 \sigma)$. The initial footprint of uncertainty (FOU) is considered as an important parameter that affects the optical axis alignment of the laser communication terminal. If the FOU is large, both the speed and probability of the acquisition are significantly reduced in this system. However, the reduction of the FOU is also limited by the precision values of the posture control, space positioning, and positioning of the platform hosting the terminal. Additionally, the FOU is affected by the pointing precision and stability of the servo turntable. All the factors that affect the FOU of the PAT system of an airborne laser communication system are listed in Table 1. 
Table 1 FOU analysis.

\begin{tabular}{|c|c|c|}
\hline Factors & Error & Comments \\
\hline Posture error & $6 \mathrm{mrad}$ & $\begin{array}{c}\text { Yaw } 4 \mathrm{mrad} \text {; roll } 3 \mathrm{mrad} \text {; pitching } \\
3 \mathrm{mrad}\end{array}$ \\
\hline GPS positioning error & $0.12 \mathrm{mrad}$ & $\begin{array}{l}\text { Height positioning error } \pm 5 \mathrm{~m} \text {, } \\
\text { positioning error } \pm 2 \mathrm{~m} \text {, shortest } \\
\text { distance } 50 \mathrm{~km}\end{array}$ \\
\hline $\begin{array}{l}\text { Pointing precision of } \\
\text { the servo turntable }\end{array}$ & $0.1 \mathrm{mrad}$ & Use high-precision sensors \\
\hline $\begin{array}{l}\text { Stability of the servo } \\
\text { turntable }\end{array}$ & $30 \mu \mathrm{rad}$ & Use active gyro stabilized servo \\
\hline Total pointing error & $6.2 \mathrm{mrad}$ & Single side, $1 \sigma$ \\
\hline
\end{tabular}

In this paper, the probability of the target appearing in the FOU is

$P_{\mathrm{cov}}=\iint_{\mathrm{FOU}} \frac{1}{\sqrt{2 \pi} \sigma} \exp \left(-\frac{\theta_{v}^{2}}{2 \sigma^{2}}\right) \cdot \frac{1}{\sqrt{2 \pi} \sigma} \exp \left(-\frac{\theta_{h}^{2}}{2 \sigma^{2}}\right) d \theta_{v} d \theta_{h}$

where $\theta_{v}$ is the angular deviation in the direction of the pitch, and $\theta_{h}$ is the angular deviation in the direction of the azimuth. The above equation can be simplified as a Rayleigh distributed amplitude and a uniformly distributed polar angle with an expectation of $1 /(2 \pi)$. The integral form (of the simplified equation) in polar coordinates is

$$
P_{U}=\int_{0}^{\frac{\mathrm{FOU}}{2}} \frac{\theta}{\sigma^{2}} \exp \left(-\frac{\theta^{2}}{2 \sigma^{2}}\right) d \theta=1-\exp \left(-\frac{\mathrm{FOU}^{2}}{8 \sigma^{2}}\right)
$$

where $\theta=\sqrt{\theta_{v}^{2}+\theta_{h}^{2}}$ is the deviation of the uncertainty angle. Usually, the uncertainty region is required to be 6 times the error of the optical axis pointing to ensure that the target's coverage probability $P_{U}$ is $98.89 \%$. Therefore, the FOU of the system is set to be $40 \mathrm{mrad}$. Errors due to the coarse and fine tracking of the system are described in Tables 2 and 3, respectively.

Table 2 Error Analysis of coarse tracking.

\begin{tabular}{ccc}
\hline Factor & $\begin{array}{c}\text { Error } \\
(\mu \mathrm{rad})\end{array}$ & Comments \\
\hline CCD measurement error & 12 & $\begin{array}{c}\text { Approximately obeys } \\
\text { Gaussian distribution }\end{array}$ \\
$\begin{array}{c}\text { Dynamic hysteresis error } \\
\text { Residual error after platform } \\
\text { vibration suppression }\end{array}$ & 25 & $\begin{array}{c}\text { Margin reserved } \\
\text { Two-axis-four-frame } \\
\text { gyro-stabilized turntable }\end{array}$ \\
Coarse tracking error & 41 & Final value \\
\hline
\end{tabular}

Table 3 Error Analysis of fine tracking.

\begin{tabular}{cc}
\hline Factor & Error $(\mu \mathrm{rad})$ \\
\hline $\begin{array}{c}\text { CCD measurement error } \\
\text { Residual after rough tracking error } \\
\text { suppression }\end{array}$ & 6 \\
Optical axis alignment error & 1 \\
Fine tracking error & 1 \\
\hline
\end{tabular}

Theoretical analysis from the above tables show that the coarse tracking error is less than $41 \mu \mathrm{rad}$ and the fine tracking error is less than $6.2 \mu \mathrm{rad}$.

\section{System performance test}

\subsection{Indoor tests}

A series of indoor tests are carried out to evaluate the dynamic tracking performance of the PAT system before any flight test. The test setup is demonstrated in Fig. 2.

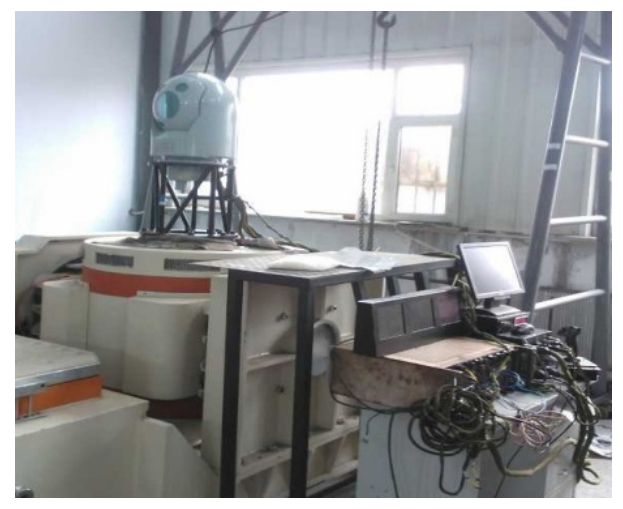

Fig. 2 Indoor test of the airborne PAT system.

The PAT system is placed on a vibrating table to simulate the vibration environment of the aircraft. Since the indoor tests are performed in a more controlled environment, the vibration table will be set to the most demanding situation according to the vibration curve of the Y12 aircraft for more realistic simulation.

\subsection{Flight tests}

After evaluating the PAT system using indoor dynamic tracking, a series of outdoor flight tests are conducted on two Y12 aircrafts to evaluate the performance of the airborne PAT system (see Fig. 3). 


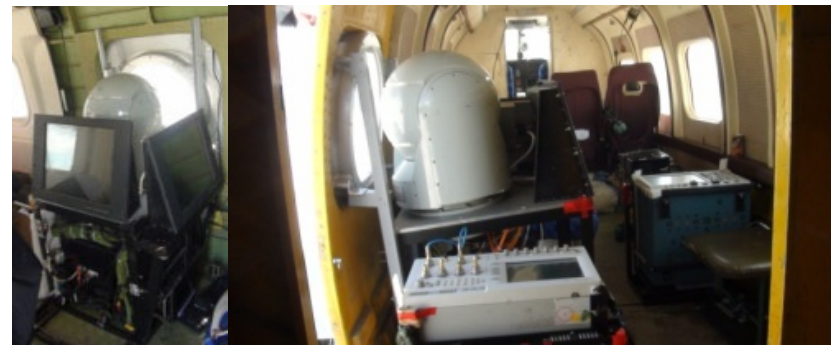

(a)

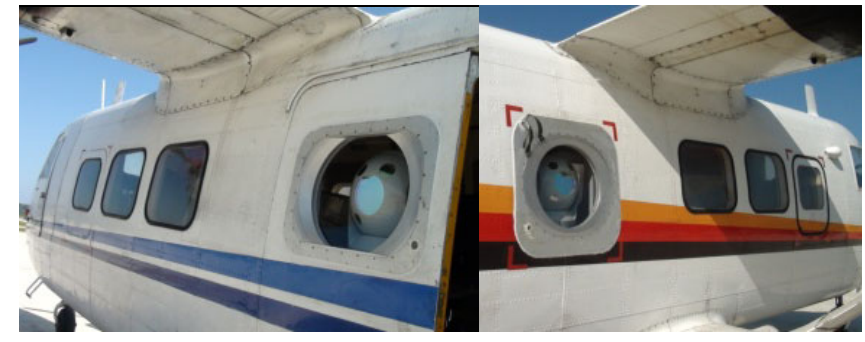

(b)

Fig. 3 Experimental setup: (a) installation of the airborne PAT system inside the cabin and (b) optical window for the laser communication test onboard two fixed-wing aircrafts.

As shown in Fig. 3, the airborne PAT test system is installed on a mounting bracket and fixed inside the cabin of the aircraft with a speed of $300 \mathrm{~km} / \mathrm{h}$.

\section{Resutls and discussion}

The results of the tracking precision test are shown in Fig. 4.

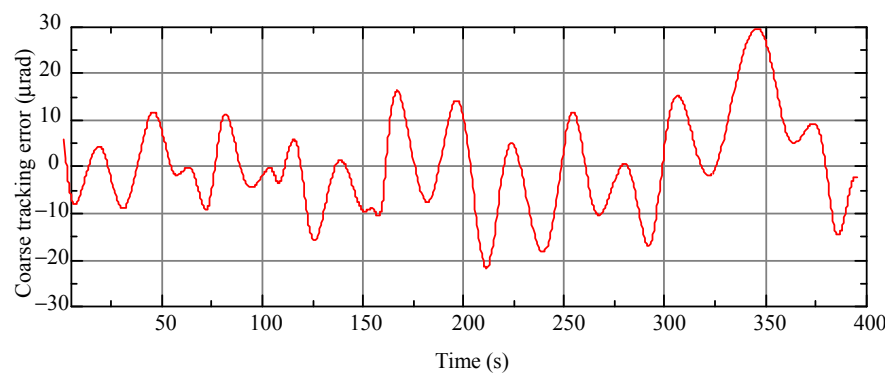

(a)

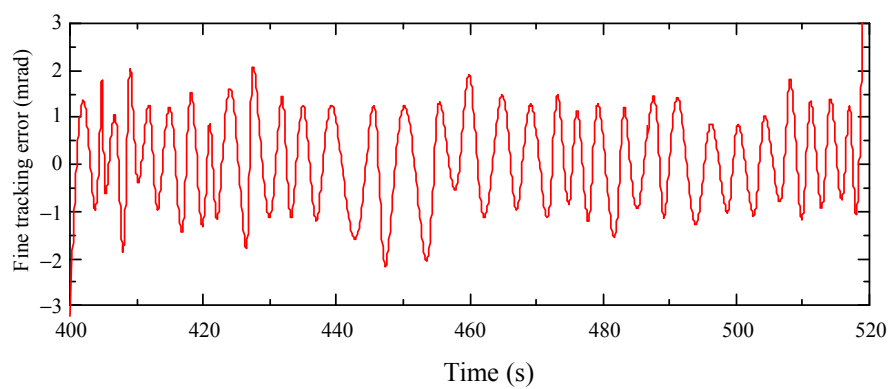

(b)

Fig. 4 Tracking precision of indoor test: (a) coarse tracking error and (b) fine tracking error. 
The results of the dynamic indoor test show that under the simulated airborne vibration condition, the PAT system can achieve a coarse tracking precision of $20 \mu \mathrm{rad} \pm 10 \mu \mathrm{rad}(1 \sigma)$, as well as a fine tracking precision of $2 \mu \mathrm{rad} \pm$
$1 \mu \operatorname{rad}(1 \sigma)$.

The angular velocity and the angular acceleration in coarse tracking and the roll angular velocity and the angular acceleration are shown in Figs. 5 and 6, respectively.

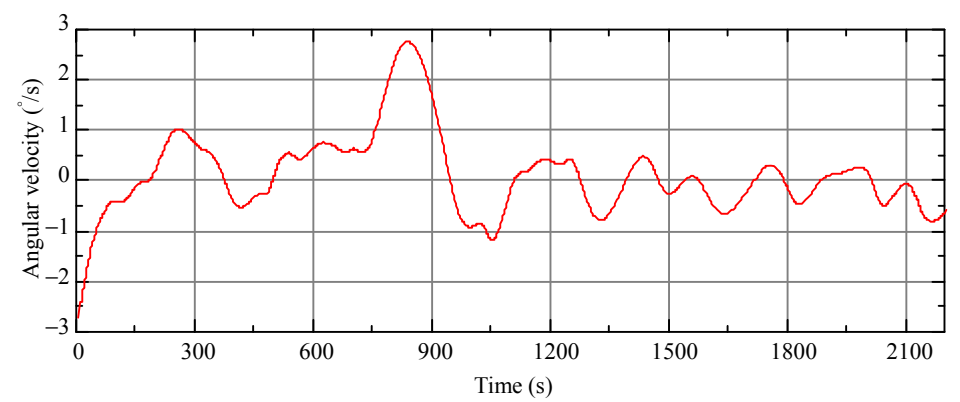

(a)

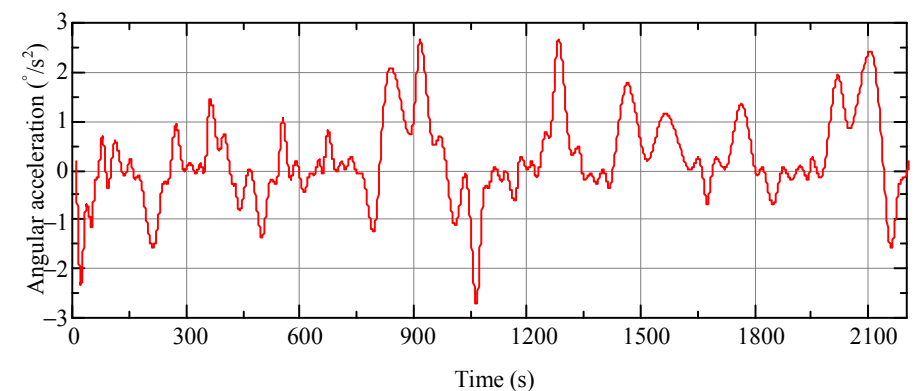

(b)

Fig. 5 Angular velocity (a) and the angular acceleration in coarse tracking (b).

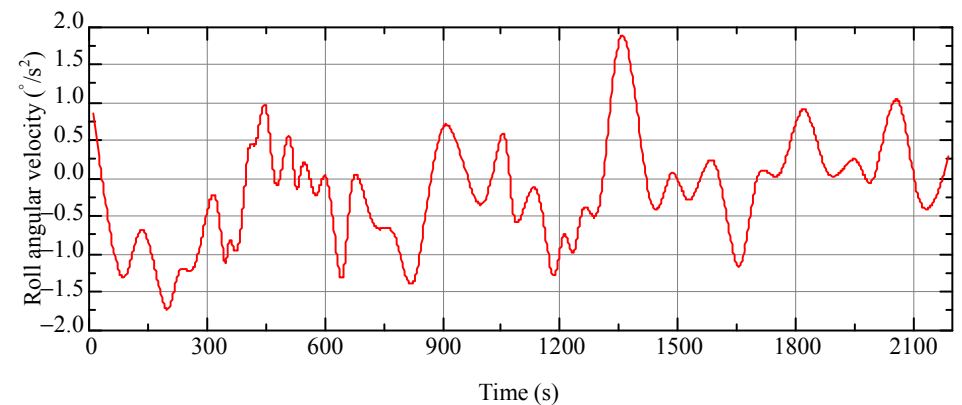

(a)

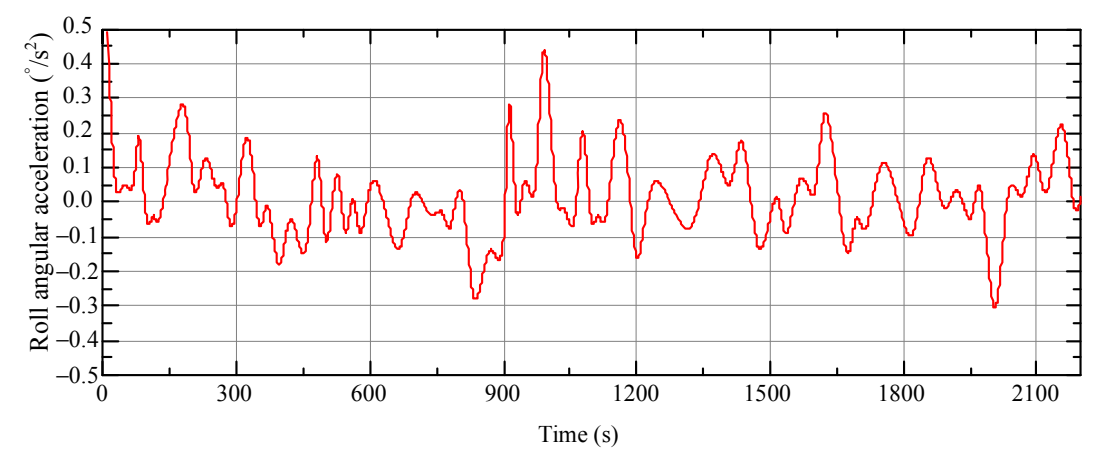

(b)

Fig. 6 Roll angular velocity (a) and the angular acceleration (b). 
As can be observed from the above figures, the posture change of the aircraft is obvious enough to identify that the maximum angular velocity of the aircraft is $2.6 \%$ and the angular acceleration is $2.5 / \mathrm{s}^{2}$. In the direction of the heading, the maximum roll angular velocity is $1.8 \%$, and the angular acceleration is $0.5^{\circ} / \mathrm{s}^{2}$, which means that a higher rotation speed is required by the turntable.

The statistical analysis of the optical terminal acquisition test results shows that out of a total of 60 acquisition attempts, 58 acquisition attempts succeed out. Therefore, the acquisition probability of the PAT system is $96.7 \%$, and the acquisition time is shown in Fig. 7.

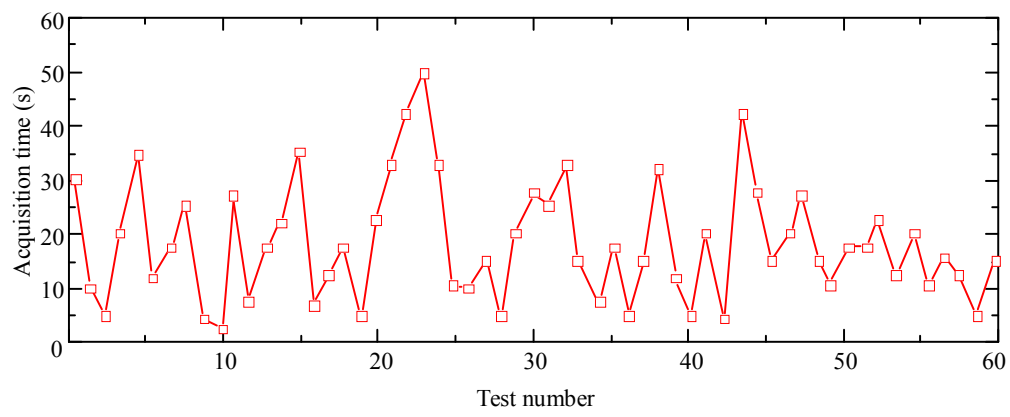

Fig. 7 Statistics of the acquisition times.

It can be seen that the average acquisition time is $18.3 \mathrm{~s}$ with the minimum time for an acquisition being $3 \mathrm{~s}$ and the maximum time being $50 \mathrm{~s}$ for the 58 successful acquisitions.

Figure 8 shows the coarse and fine tracking errors of the PAT system under the test flight conditions.

It can be seen that the coarse tracking error $(1 \sigma)$ is $8.68 \mu \mathrm{rad}$ and the fine tracking error is $8.19 \mu \mathrm{rad}$.
Compared with the indoor tests, the coarse tracking error is decreased almost $22 \mu \mathrm{rad}$ which means the capability of the PAT system is much improved since the indoor tests are performed in a nearly the same or even severe situation as the flight tests. Also, the variance of the fine tracking error of the flight tests is much smaller although they basically share the same maximum.

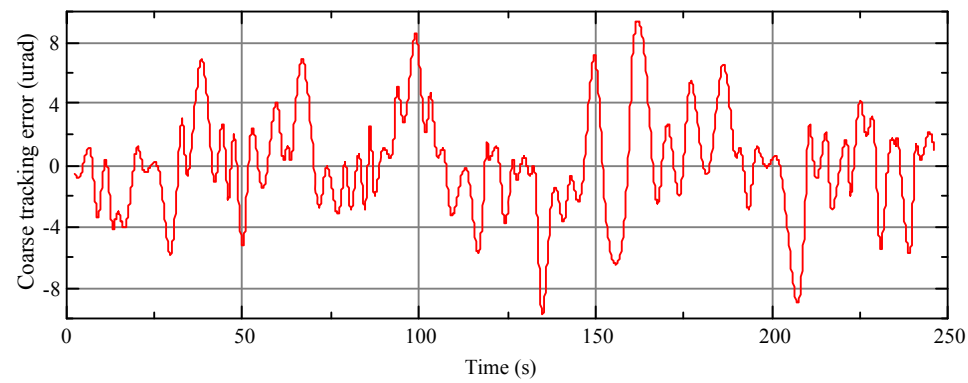

(a)

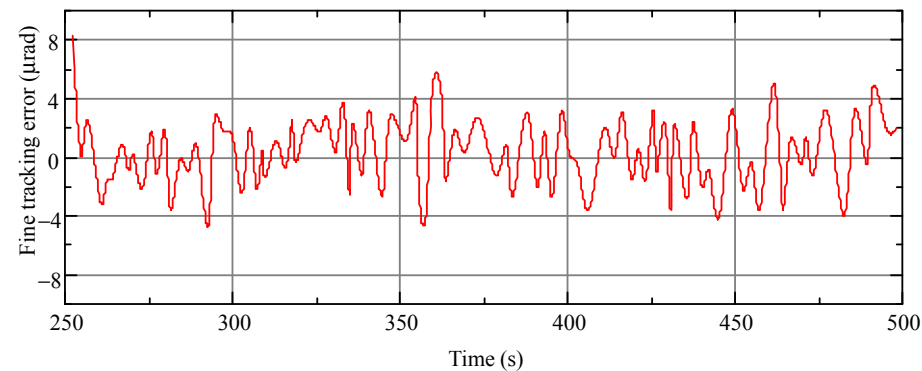

(b)

Fig. 8 Flight tracking precision test: (a) coarse tracking error and (b) fine tracking error. 
After achieving stable tracking, the quality of the communication and the power distribution of the received light are measured by maintaining a communication distance of $144 \mathrm{~km}$. The results of the power distribution measurements are shown in Fig. 9.

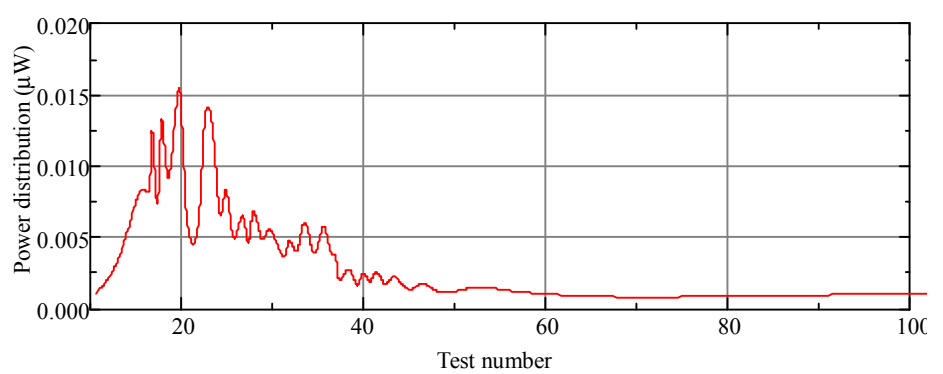

Fig. 9 Power distribution of the received light during the communication.

It can be observed that the coarse-fine composite tracking system provides a reasonably high axis pointing precision with a relatively concentrated energy distribution and a low jitter variance around $0.0001 \mu \mathrm{W}$. This may be a result of the improved optical axis pointing accuracy supplied by the proposed PAT system, which is also demonstrated that this PAT system is capable of ensuring a desired communication quality.

\section{Conclusions}

In this study, the key parameters of the proposed PAT system are studied and analyzed with an aircraft-based platform for free-space laser communications. The PAT system consisting of a biaxial-quad-frame coarse tracking module and a fine tracking unit based on piezo-ceramic galvanometer is adopted in this paper. The system is evaluated through long-distance $(10 \mathrm{~km}-144 \mathrm{~km})$ acquisition and tracking experiments, and performed on two fixed-wing Y12 aircrafts. This is the first time such an experiment is carried out in China. Experimental results show that the coarse tracking precision of the proposed PAT system is less than $10 \mu \mathrm{rad}(1 \sigma)$, and the fine tracking precision is less than $8 \mu \mathrm{rad}(1 \sigma)$ during the flights. A higher communication rate of $2.5 \mathrm{Gbps}$ with a low bit error rate around $10^{-7}$ is promoted to verify that the PAT system proposed in this paper is capable of providing the desired pointing, acquisition and tracking accuracy which is essential for a long-distance and high-speed laser communications.

\section{Acknowledgment}

This project was supported by the National Natural Science Foundation of China (Grant No. 51505087). Also, we thank the Collaborative Innovation Center of High-End Equipment Manufacturing in Fujian Province of China for applying the experimental field.

Open Access This article is distributed under the terms of the Creative Commons Attribution 4.0 International License (http://creativecommons.org/licenses/by/4.0/), which permits unrestricted use, distribution, and reproduction in any medium, provided you give appropriate credit to the original author(s) and the source, provide a link to the Creative Commons license, and indicate if changes were made.

\section{References}

[1] J. C. Juarez, D. W. Young, J. E. Sluz, and D. H. Hughes, "Free-space optical channel propagation tests over a 147 km link," SPIE, 2011, 8038(1): 80380B-1-80380B-10.

[2] T. M. Fletcher, J. Cunningham, D. Baber, D. Wickholm, T. Goode, B. Gaughan, et al., "Observations of atmospheric effects for FALCON laser communication system flight test," SPIE, 2011, 8038(1): 80380F-1-80380F-12.

[3] M. Q. Li, "Study on the simulation of the APT rough tracing system based on the predictive control technology," Computer and Information Science, 2012, 5(2): 45-52.

[4] J. F. Sun, M. J. Yun, L. Y. Wan, Z. Luan, and L. R. Liu, 
"Satellite trajectory simulator for inter-satellite laser communication system APT tests," SPIE, 2005, 6024: 60241T-1-60241T-5.

[5] M. Li, P. F. Lu, Z. Y. Yu, Y. M. Liu, L. D. Zhang, and C. H. Yang, "General model on polarization compensation in satellite-to-ground quantum communication," Optical Engineering, 2013, 52(4): 045001-1-045001-6.

[6] Y. Lei, Y. Bai, and Z. Xu, "Sensor-based inspection of the formation accuracy in ultra-precision grinding (UPG) of aspheric surface considering the chatter vibration," Photonic Sensors, 2018, 8(2): 97-102.

[7] T. Shang, J. J. Jia, and X. Wang, “Analysis and design of a multi-transceiver optical cylinder antenna for mobile free space optical communication," Optics \& Laser Technology, 2012, 44(8): 2384-2392.

[8] H. B. Huang, W. R. Jiang, Y. Ai, and T. Zuo, "Adopting gradient vector and particle filter in APT for space optical communication," in Proceeding of First International Conference on Intelligent Networks and Intelligent Systems, Wuhan, China, 2008, pp. 301-304.

[9] D. Ruffner, "Optical forces in complex beams of light," Ph.D. dissertation, New York University, New York, USA, 2015.

[10] A. Ashlaghi, "100 GBPS orthogonal frequency division multiplexing optical fiber communication network," Master dissertation, California State University, California, USA, 2015.

[11] S. Arnon and N. S. Kopeika, "Adaptive optical transmitter and receiver for space communication through thin clouds," Applied Optics, 1997, 36(9): 1987-1993.

[12] Y. Lei, Z. J. Xu, and L. Z. Zhang, "Experimental study on thermal uniformity of optical transmitter and receiver on near space," Experimental Thermal \& Fluid Science, 2011, 35(7): 1463-1472.

[13] T. Luo and H. U. Yu, "Design and realization of laser beam acquisition, pointing and tracking of ground demonstration system for free space optical communication," Journal of Applied Optics, 2002, 23(2): 14-17.

[14] J. Cunningham, D. Grinch, and D. Fisher, "Acquisition, pointing, and tracking architecture for laser communication," U.S. Patent 7609972 B2, Oct. 27, 2009.

[15] T. H. Ho, S. D. Milner, and C. C. Davis, "Fully optical real-time pointing, acquisition, and tracking system for free space optical link," SPIE, 2005, 5712: 81-92.

[16] T. H. Ho, S. Trisno, I. I. Smolyaninov, S. D. Milner, and C. C. Davis, "Studies of pointing, acquisition, and tracking of agile optical wireless transceivers for free-space optical communication networks," SPIE, 2004, 5237: 147-158. 